\title{
Chiral isothiourea-catalyzed kinetic resolution of 4-hydroxy[2.2]paracyclophane
}

\author{
David Weinzierl and Mario Waser ${ }^{*}$
}

\section{Letter}

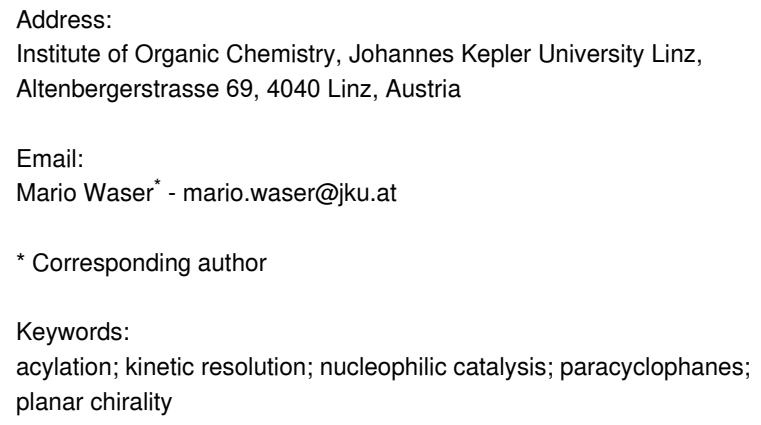

Beilstein J. Org. Chem. 2021, 17, 800-804. https://doi.org/10.3762/bjoc. 17.68

Received: 24 February 2021

Accepted: 01 April 2021

Published: 08 April 2021

This article is part of the thematic issue "New advances in asymmetric organocatalysis" and is dedicated to Prof. Dr. Norbert Müller, who has been a supportive mentor and good friend over all the years, on the occasion of his 65 th birthday.

Guest Editor: R. Šebesta

(C) 2021 Weinzierl and Waser; licensee Beilstein-Institut. License and terms: see end of document.

\section{Abstract}

We herein report a method for the kinetic resolution of racemic 4-hydroxy[2.2]paracyclophane by means of a chiral isothioureacatalyzed acylation with isobutyric anhydride. This protocol allows for a reasonable synthetically useful $s$-factor of 20 and provides a novel entry to obtain this interesting planar chiral motive in an enantioenriched manner.

\section{Introduction}

Substituted [2.2]paracyclophanes are fascinating planar chiral molecules [1-12] which have been systematically investigated since Brown and Farthing discovered the formation of the unsubstituted and achiral parent [2.2]paracyclophane (1) via gas phase pyrolysis of para-xylene in 1949 [5]. Over the years, these compounds established themselves as a unique class of "bent and battered" [6] strained molecules with remarkable chemical and physical properties [1-4,7-9]. Besides their potential applications in material and polymer chemistry [1,2,7-9], these planar chiral molecules have been very successfully used in asymmetric catalysis [3,4,10-12]. Accordingly, the development of methods for the asymmetric synthesis of enantiomeri- cally pure, or at least enantiomerically enriched, derivatives that can be utilized as building blocks for more demanding ligands and catalysts became a task of high importance. Thus, several strategies to access enantioenriched [2.2]paracyclophanes have been reported, either relying on classical resolution approaches or, more recently, making use of asymmetric catalysis to carry out kinetic resolutions of easily accessed racemic precursors [3,4,13-15]. 4-Hydroxy[2.2]paracyclophane (2) is one of the commonly used building blocks, which is easily accessible in a racemic manner starting from $\mathbf{1}$ according to nowadays wellestablished procedures [16-18]. Over the last decades, it was shown that enantioenriched $\mathbf{2}$ may serve as a valuable building 
block to access more advanced chiral cyclophane ligands and catalysts $[3,4,19-22]$ and therefore its asymmetric synthesis became an important task [3,4,18-27]. Several strategies to access 2 in an enantioenriched fashion have been developed. One commonly used method relies on the resolution of 4-formyl[2.2]paracyclophane via formation of a chiral Schiff base first, followed by a subsequent Dakin-type oxidation to alcohol 2 [18]. Alternatively, the direct resolution of $\mathrm{rac}-\mathbf{2}$ via transformation into diastereomers by esterification with chiral acid chlorides $[19,20]$ as well as the kinetic resolution (KR) of racemic esters of $\mathbf{2}$ via an enzymatic hydrolysis [25-27] were very successfully used to access enantioenriched $\mathbf{2}$. Recently, Akiyama and co-workers reported the kinetic resolution of $\mathrm{rac}$ PHANOL (4,12-dihydroxy[2.2]paracyclophane) by means of a chiral phosphoric acid-catalyzed esterification with achiral anhydrides [28]. This method allowed for high $s$-factors but was unfortunately not satisfyingly applicable to rac-4hydroxy[2.2]paracyclophane (rac-2) [28].

Considering the interest in compound $\mathbf{2}$, we thus thought about developing an alternative organocatalytic kinetic resolution protocol to control the esterification of rac-2. Chiral isothioureas (ITUs) emerged as easily available and powerful catalysts for numerous applications [29-32] and have been very successfully used for the kinetic resolution of different racemic alcohols [33-37]. Inspired by this unique catalysis potential, we therefore became interested in testing those chiral catalysts for the, to the best of our knowledge, so far not investigated acylative kinetic resolution of 4-hydroxy[2.2]paracyclophane (2, Scheme 1).

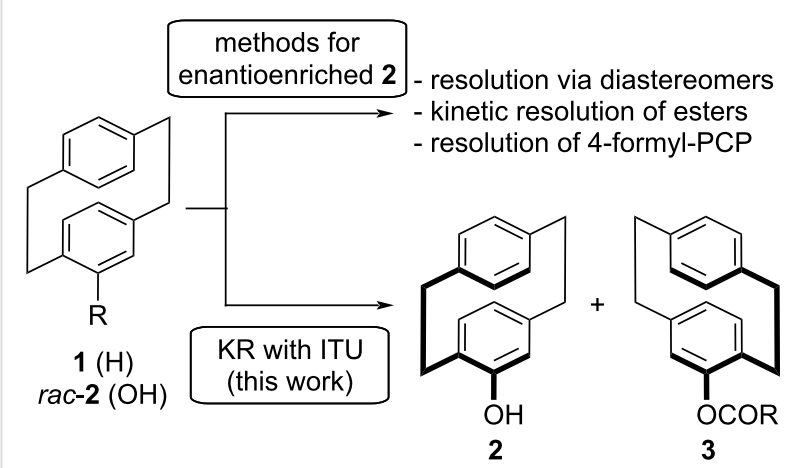

Scheme 1: Overview about established methods to access enantioenriched 2 and the herein investigated kinetic resolution (KR) with chiral isothiourea (ITU) catalysts.

\section{Results and Discussion}

BTM (ITU 1 [33]) and HyperBTM (ITU 2 [38]) are amongst the most commonly used chiral ITUs and these nowadays commercially available catalysts were used to optimize the resolution of rac-2 with isobutyric anhydride (4a) (Table 1 gives an overview of the most significant results obtained in this screening). Anhydride 4a was chosen in a first instance as it proved successful in previous acylative resolutions reported by others $[28,33,34,36,37]$ but we later on also tested other anhydrides and acid chlorides (vide infra, Scheme 2). First experiments with $10 \mathrm{~mol} \% \mathrm{BTM}$ (ITU 1) carried out in $\mathrm{CHCl}_{3}$ or toluene at room temperature (Table 1, entries 1 and 2) proved the general feasibility of this concept, resulting in $s$-factors around 6. When lowering the temperature, a slight improvement could be achieved at $-15{ }^{\circ} \mathrm{C}$ (Table 1 , entry 3 ) but unfortunately ITU 1 performed less selective at $-78{ }^{\circ} \mathrm{C}$ (Table 1 , entry 4 ). Instead, (2S,3R)-HyperBTM (ITU 2) resulted in an enhanced selectivity with $s=14.5$ at $-78{ }^{\circ} \mathrm{C}$ but conversion was relatively slow (Table 1, entry 5). Gratefully however, the obtained $s$-factor was almost the same at $-40{ }^{\circ} \mathrm{C}$ and a reasonable conversion of around $30 \%$ could be observed after $4 \mathrm{~h}$ reaction time (Table 1, entry 6). Varying solvent and concentration at $-40{ }^{\circ} \mathrm{C}$ next showed that toluene allows for higher selectivities than $\mathrm{CHCl}_{3}$ (compare Table 1, entries 6 and 7), while the use of other solvents like $\mathrm{CH}_{2} \mathrm{Cl}_{2}$ and THF resulted in almost no product formation and no reasonable selectivities (not mentioned in Table 1). In addition, higher concentrations lead to notably lower selectivities (Table 1, entry 9), while more diluted conditions did not allow for a significant improvement of the $s$-factor anymore (Table 1 , entry 8 ). Lowering the catalyst loading from 10 to $5 \mathrm{~mol} \%$ allowed for a similar conversion, but resulted in a slightly reduced selectivity (Table 1 , entry 10 ).

At this point, we decided to screen other anhydrides and acid chlorides 4, but, as outlined in Scheme 2, the initially used isobutyric anhydride $\mathbf{4 a}$ clearly outperformed its analogous acid chloride $\mathbf{4 b}$, as well as the other derivatives $\mathbf{4 c - f}$.

Finally, the resolution of $\mathrm{rac}-2$ was run for $22 \mathrm{~h}$ in the presence of $10 \mathrm{~mol} \%$ HyperBTM (ITU 2) with 1.1 equivalents of anhydride $4 \mathbf{a}$ (instead of the previously used 0.6 equiv; Table 1 , entry 11). Under these conditions it was possible to achieve a conversion of slightly above $50 \%$ combined with good enantioselectivities for both, the recovered alcohol $\mathbf{2}$ and the ester $\mathbf{3 a}$ $(s=20)$. With these optimum conditions the resolution was also successfully carried out on $1 \mathrm{mmol}$ scale, resulting in an identical conversion and $s$-factor $(s=20 ; C=57 \%)$ and allowing for the isolation of $\left(R_{\mathrm{p}}\right)-\mathbf{2}$ in $39 \%$ yield $\left(94 \%\right.$ ee) and $\left(S_{\mathrm{p}}\right)-\mathbf{3 a}$ in $53 \%$ yield ( $71 \%$ ee) (Table 1 , entry 11$)$. Mechanistically, this resolution process should proceed via the well-understood formation of a chiral acyl-transfer species between the isothiourea catalyst ITU 2 and the anhydride 4a [33-37], which then allows for the resolution of the enantiomers of alcohol 2. Unfortunately, however, the true nature of this enantiodiscriminating step has not yet been elucidated and will require detailed computational studies. 
Table 1: Identification of the optimum catalyst and best conditions for the resolution of rac-2 with anhydride $4 a^{a}$.<smiles>O=C(O)c1ccc(CCc2ccc(CCc3ccccc3)cc2)cc1</smiles><smiles>CC(C)C(=O)OC(=O)C(C)C</smiles>
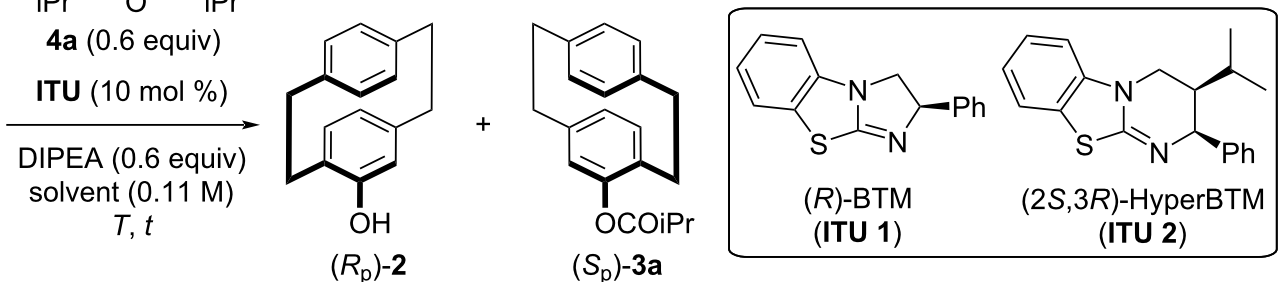

$\left(S_{\mathrm{p}}\right)-3 \mathrm{a}$

\begin{tabular}{lllllllll}
\hline Entry & ITU & Solvent & $T\left[{ }^{\circ} \mathrm{C}\right]$ & $t[\mathrm{~h}]$ & Conv. $(\mathbf{C})[\%]^{\mathrm{b}}$ & ee $(\mathbf{2})[\%]^{\mathrm{c}, \mathrm{d}}$ & ee $(\mathbf{3 a})[\%]^{\mathrm{c}}$ & $s^{\mathrm{e}}$ \\
\hline 1 & ITU 1 & $\mathrm{CHCl}_{3}$ & 25 & 1 & 41 & 42 & 60 & 6 \\
2 & ITU 1 & toluene & 25 & 1 & 38 & 39 & 64 & 6.5 \\
3 & ITU 1 & toluene & -15 & 1 & 34 & 38 & 74 & 10 \\
4 & ITU 1 & toluene & -78 & 1 & 15 & 13 & 74 & 7.5 \\
5 & ITU 2 & toluene & -78 & 1 & 16 & 16 & 85 & 14.5 \\
6 & ITU 2 & toluene & -40 & 4 & 33 & 40 & 81 & 14 \\
7 & ITU 2 & CHCl & -40 & 4 & 45 & 55 & 67 & 9 \\
8 & ITU 2 & toluene (0.055 M) & -40 & 4 & 30 & 35 & 82 & 14.5 \\
9 & ITU 2 & toluene (0.22 M) & -40 & 4 & 36 & 32 & 75 & 9.5 \\
$10^{f}$ & ITU 2 & toluene & -40 & 4 & 30 & 34 & 79 & 12 \\
119 & ITU 2 & toluene & -40 & 22 & 57 & $94(39 \%)^{\mathrm{h}}$ & $71(53 \%)^{\mathrm{h}}$ & 20 \\
\hline
\end{tabular}

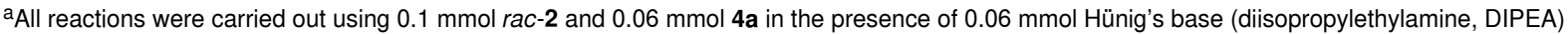
and $10 \mathrm{~mol} \%$ ITU in the indicated solvent $(0.11 \mathrm{M}$ with respect to 2$)$ unless otherwise stated; ${ }^{b}$ determined by ${ }^{1} \mathrm{H}$ NMR of the crude product; isolated yields of $\mathbf{2}$ and $\mathbf{3}$ were almost quantitative in all cases; ' ${ }^{\circ}$ determined by HPLC using a chiral stationary phase; dabsolute configuration of recovered $\mathbf{2}$

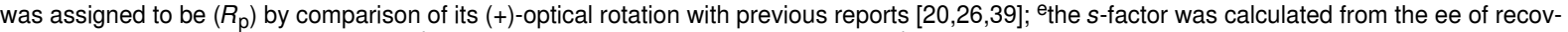

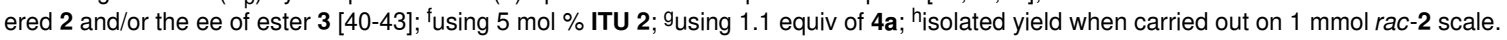

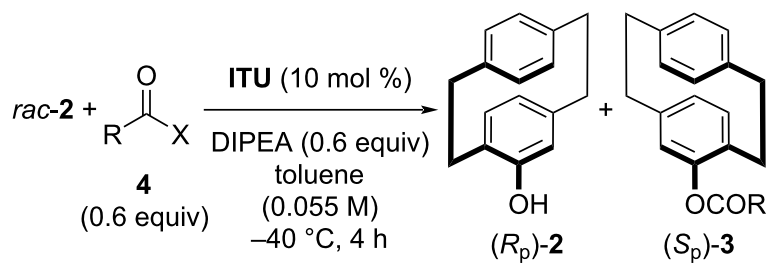

iPr

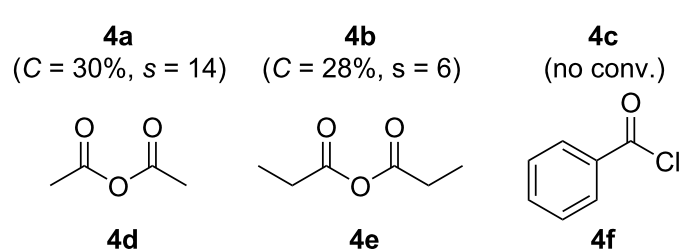

$(C=45 \%, s=2.5) \quad(C=38 \%, s=4.5)(C=28 \%$, racemic $)$

Scheme 2: Use of alternative acylating agents 4 for the kinetic resolution of rac-2.

\section{Conclusion}

In conclusion, we identified conditions that allow for the kinetic resolution of racemic 4-hydroxy[2.2]paracyclophane (2) by means of an acylation with isobutyric anhydride (4a) in the presence of the chiral isothiourea catalyst HyperBTM (ITU 2). The reaction can be carried out with an $s$-factor around 20 and allows for the isolation of recovered $\left(R_{\mathrm{p}}\right)-\mathbf{2}$ and ester $\left(S_{\mathrm{p}}\right)-\mathbf{3 a}$ with reasonable enantiomeric excesses around $90 \%$, depending on the conversion. These two compounds can easily be separated by silica gel column chromatography in almost quantitative yields, thus providing a novel entry to obtain these interesting planar chiral motives in an enantioenriched manner.

\section{Experimental \\ General details}

${ }^{1} \mathrm{H}$ - and ${ }^{13} \mathrm{C}$ NMR spectra were recorded on a Bruker Avance III $300 \mathrm{MHz}$ spectrometer with a broad band observe probe and a sample changer for 16 samples. NMR spectra were referenced on the solvent peak and chemical shifts are given in ppm.

High-resolution mass spectra were obtained using a Thermo Fisher Scientific LTQ Orbitrap XL with an Ion Max API Source. Analyses were made in the positive ionization mode if not otherwise stated. HPLC was performed using a Thermo Scientific Dionex Ultimate 3000 system with diode array 
detector with a CHIRAL ART Cellulose-SB stationary phase. Optical rotations were recorded on a Schmidt + Haensch Polarimeter Model UniPol L1000 at $589 \mathrm{~nm}$.

All chemicals were purchased from commercial suppliers and used without further purification unless otherwise stated. $r a c-2$ was prepared from $\mathbf{1}$ according to a previously published procedure [16].

\section{Optimized procedure for the KR of rac-2}

Racemic 4-hydroxy[2.2]paracyclophane (rac-2; $250 \mathrm{mg}$; $1.115 \mathrm{mmol}$ ) and HyperBTM (ITU 2; $35 \mathrm{mg} ; 10 \mathrm{~mol} \%$ ) were dissolved in dry toluene $(10 \mathrm{~mL})$ in a Schlenk flask (Ar atmosphere), followed by the addition of Hünig's base (DIPEA; $118 \mu \mathrm{L} ; 0.67 \mathrm{mmol} ; 0.6$ equiv). The solution was then cooled to $-40{ }^{\circ} \mathrm{C}$ and isobutyric anhydride $(4 \mathbf{a} ; 208 \mu \mathrm{L} ; 1.226 \mathrm{mmol}$; 1.1 equiv) was added and the mixture was stirred at $-40{ }^{\circ} \mathrm{C}$ for $22 \mathrm{~h}$. The reaction was quenched by addition of $\mathrm{MeOH}$. The crude product was filtered over $\mathrm{Na}_{2} \mathrm{SO}_{4}$ and the solvent removed in vacuum. Recovered alcohol $\mathbf{2}$ and ester 3a were separated by silica gel column chromatography (heptanes/ethyl acetate $10: 1)$, yielding $\left(S_{\mathrm{p}}\right)$-3a in 53\% (175 mg) and $\left(R_{\mathrm{p}}\right)-\mathbf{2}$ in $43 \%$ (98 mg) (39\%).

$\left(R_{\mathrm{p}}\right)$-2a: Analytical data match those reported in literature [1820,26,28,39]. TLC (heptanes/ethyl acetate 10:1; $R_{\mathrm{f}}=0.11$ ). $[\alpha]_{\mathrm{D}}^{24} 14.1$ (c 1, $\mathrm{CH}_{2} \mathrm{Cl}_{2}, 92 \%$ ee) and 12.1 (c $1, \mathrm{CHCl}_{3}, 92 \%$ ee); ${ }^{1} \mathrm{H}$ NMR (300 MHz, $\left.\mathrm{CDCl}_{3}, 298.0 \mathrm{~K}\right) \mathrm{\delta} / \mathrm{ppm} 7.00(\mathrm{dd}, J=$ $8,1.8 \mathrm{~Hz}, 1 \mathrm{H}), 6.55(\mathrm{dd}, J=8,1.8 \mathrm{~Hz}, 1 \mathrm{H}), 6.45(\mathrm{dd}, J=8$, $1.8 \mathrm{~Hz}, 1 \mathrm{H}), 6.41-6.37(\mathrm{~m}, 2 \mathrm{H}), 6.26(\mathrm{dd}, J=8,1.8 \mathrm{~Hz}, 1 \mathrm{H})$, $5.54(\mathrm{~d}, J=1.6 \mathrm{~Hz}, 1 \mathrm{H}), 4.42(\mathrm{~s}, 1 \mathrm{H}), 3.37-3.29(\mathrm{~m}, 1 \mathrm{H})$, 3.14-3.02 (m, 4H), 2.98-2.85 (m, 2H), 2.71-2.60 (m, 1H); ${ }^{13} \mathrm{C} \mathrm{NMR}\left(75 \mathrm{MHz}, \mathrm{CDCl}_{3}, 298.0 \mathrm{~K}\right) \mathrm{\delta} / \mathrm{ppm} 153.8$ (1C, CAr), 142.1 (1C, CAr), 139.8 (1C, CAr), 139.0 (1C, CAr), 135.6 (1C, CAr), 133.8 (1C, CAr), 132.9 (1C, CAr), 132.0 (1C, CAr), 128.1 (1C, CAr), 125.6 (1C, CAr), 125.2 (1C, CAr), 122.7 (1C, CAr), $35.4\left(1 \mathrm{C},-\mathrm{CH}_{2}\right), 34.9\left(1 \mathrm{C},-\mathrm{CH}_{2}\right), 34.0\left(1 \mathrm{C},-\mathrm{CH}_{2}\right), 32.2$ (1C, $-\mathrm{CH}_{2}$ ); HRMS (ESI) $\mathrm{m} / \mathrm{z}$ : calcd for $\left[\mathrm{C}_{16} \mathrm{H}_{16} \mathrm{O}+\mathrm{H}\right]^{+}$, 225.1274; found, 225.1280, HPLC: YMC Chiral ART Cellulose-SB, $n$-hexane/iPrOH $3: 1,1 \mathrm{~mL} / \mathrm{min}, 10{ }^{\circ} \mathrm{C} ; t_{\mathrm{R}}=6.4 \mathrm{~min}$ [S $S_{\mathrm{p}}$; minor], $7.2 \min \left[R_{\mathrm{p}}\right.$; major].

$\left(S_{\mathrm{p}}\right)$-3a: Analytical data match those reported in literature [28]. TLC (heptanes/ethyl acetate $\left.10: 1 ; R_{\mathrm{f}}=0.33\right)$. $[\alpha]_{\mathrm{D}}{ }^{24} 27.5(c 1.0$, $\mathrm{CHCl}_{3}, 82 \%$ ee); mp 80-82 ${ }^{\circ} \mathrm{C}$; ${ }^{1} \mathrm{H}$ NMR (300 MHz, $\mathrm{CDCl}_{3}$, $298.0 \mathrm{~K}) \delta / \mathrm{ppm} 6.91(\mathrm{dd}, J=7.8,1.8 \mathrm{~Hz}, 1 \mathrm{H}), 6.56-6.43(\mathrm{~m}$, $5 \mathrm{H}), 6.00(\mathrm{~d}, J=1.7 \mathrm{~Hz}, 1 \mathrm{H}), 3.17-2.94(\mathrm{~m}, 7 \mathrm{H}), 2.93-2.79(\mathrm{~m}$, $1 \mathrm{H}), 2.73-2.64(\mathrm{~m}, 1 \mathrm{H}), 1.42(\mathrm{~d}, J=7 \mathrm{~Hz}, 3 \mathrm{H}), 1.38$ (d, $J=$ $7 \mathrm{~Hz}, 3 \mathrm{H}) ;{ }^{13} \mathrm{C} \mathrm{NMR}\left(75 \mathrm{MHz}, \mathrm{CDCl}_{3}, 298.0 \mathrm{~K}\right)$ 8/ppm 174.8 (1C, C=O), 149.1 (1C, CAr), 141.7 (1C, CAr), 139.6 (1C, CAr), 139.3 (1C, CAr), 135.4 (1C, CAr), 133.5 (1C, CAr), 133.1 (1C,
CAr), 132.3 (1C, CAr), 131.1 (1C, CAr), 130.1 (1C, CAr), 129.6 (1C, CAr), 128.2 (1C, CAr), $35.4\left(1 \mathrm{C},-\mathrm{CH}_{2}\right), 35.0$ (1C, $\left.-\mathrm{CH}_{2}\right), 34.4\left(2 \mathrm{C},-\mathrm{CH},-\mathrm{CH}_{2}\right), 31.8\left(1 \mathrm{C},-\mathrm{CH}_{2}\right), 19.4(1 \mathrm{C}$, $\left.-\mathrm{CH}_{3}\right), 19.1\left(1 \mathrm{C},-\mathrm{CH}_{3}\right)$; HRMS (ESI) $m / z$ : calcd for $\left[\mathrm{C}_{20} \mathrm{H}_{22} \mathrm{O}_{2}\right.$ $\left.+\mathrm{NH}_{4}\right]^{+}, 312.1958$; found, 312.1958, HPLC: YMC Chiral ART Cellulose-SB, $n$-hexane/iPrOH $3: 1,1 \mathrm{~mL} / \mathrm{min}, 10^{\circ} \mathrm{C} ; t_{\mathrm{R}}=$ $7.3 \min \left[R_{\mathrm{p}} ;\right.$ minor], $8.4 \min \left[S_{\mathrm{p}}\right.$; major].

\section{Supporting Information}

\section{Supporting Information File 1}

Copies of NMR spectra and HPLC chromatograms as well as analytical data of esters $\mathbf{3}$ obtained with the alternative acyl-transfer reagents 4 .

[https://www.beilstein-journals.org/bjoc/content/ supplementary/1860-5397-17-68-S1.pdf]

\section{Acknowledgements}

We are grateful to Thomas Bögl (Institute of Analytical Chemistry, JKU Linz) for support with HRMS analysis.

\section{Funding}

This work was generously supported by the Austrian Science Funds (FWF): Project No. P31784. The used NMR spectrometers were acquired in collaboration with the University of South Bohemia (CZ) with financial support from the European Union through the EFRE INTERREG IV ETC-AT-CZ program (project M00146, "RERI-uasb").

\section{ORCID ${ }^{\circledR}$ iDs}

Mario Waser - https://orcid.org/0000-0002-8421-8642

\section{Preprint}

A non-peer-reviewed version of this article has been previously published as a preprint: https://doi.org/10.3762/bxiv.2021.14.v1

\section{References}

1. Gleiter, R.; Hopf, H., Eds. Modern Cyclophane Chemistry; Wiley-VCH: Weinheim, Germany, 2004. doi:10.1002/3527603964

2. Hopf, H. Angew. Chem., Int. Ed. 2008, 47, 9808-9812. doi:10.1002/anie.200800969

3. Hassan, Z.; Spuling, E.; Knoll, D. M.; Lahann, J.; Bräse, S. Chem. Soc. Rev. 2018, 47, 6947-6963. doi:10.1039/c7cs00803a

4. Hassan, Z.; Spuling, E.; Knoll, D. M.; Bräse, S. Angew. Chem., Int. Ed. 2020, 59, 2156-2170. doi:10.1002/anie.201904863

5. Brown, C. J.; Farthing, A. C. Nature 1949, 164, 915-916. doi:10.1038/164915b0

6. Cram, D. J.; Cram, J. M. Acc. Chem. Res. 1971, 4, 204-213. doi:10.1021/ar50042a003

7. Elacqua, E.; MacGillivray, L. R. Eur. J. Org. Chem. 2010, 6883-6894. doi:10.1002/ejoc.201000930 
8. Marrocchi, A.; Tomasi, I.; Vaccaro, L. Isr. J. Chem. 2012, 52, 41-52. doi:10.1002/ijch.201100091

9. Mori, T.; Inoue, Y. Top. Curr. Chem. 2010, 298, 99-128.

10. Rowlands, G. J. Isr. J. Chem. 2012, 52, 60-75. doi:10.1002/ijch.201100098

11. Paradies, J. Synthesis 2011, 3749-3766. doi:10.1055/s-0031-1289296

12. Gibson, S. E.; Knight, J. D. Org. Biomol. Chem. 2003, 1, 1256-1269. doi:10.1039/b300717k

13. Kotha, S.; Shirbhate, M. E.; Waghule, G. T. Beilstein J. Org. Chem. 2015, 11, 1274-1331. doi:10.3762/bjoc.11.142

14. Rowlands, G. J. Org. Biomol. Chem. 2008, 6, 1527-1534. doi:10.1039/b800698a

15. Tanaka, K. Bull. Chem. Soc. Jpn. 2018, 91, 187-194. doi:10.1246/bcsj.20170346

16. Krohn, K.; Rieger, H.; Hopf, H.; Barrett, D. Chem. Ber. 1990, 123, 1729-1732. doi:10.1002/cber.19901230824

17. Kane, V. V.; Gerdes, A.; Grahn, W.; Ernst, L.; Dix, I.; Jones, P. G.; Hopf, H. Tetrahedron Lett. 2001, 42, 373-376. doi:10.1016/s0040-4039(00)01992-4

18. Friedmann, C. J.; Ay, S.; Bräse, S. J. Org. Chem. 2010, 75 , 4612-4614. doi:10.1021/jo100468s

19. Rozenberg, V.; Danilova, T.; Sergeeva, E.; Vorontsov, E.; Starikova, Z.; Korlyukov, A.; Hopf, H. Eur. J. Org. Chem. 2002, 468-477. doi:10.1002/1099-0690(20022)2002:3<468::aid-ejoc468>3.0.co;2-3

20. Zhang, T.-Z.; Dai, L.-X.; Hou, X.-L. Tetrahedron: Asymmetry 2007, 18, 251-259. doi:10.1016/j.tetasy.2007.01.020

21. Vorontsova, N. V.; Zhuravsky, R. P.; Sergeeva, E. V.; Vorontsov, E. V.; Starikova, Z. A.; Rozenberg, V. I. Russ. Chem. Bull. 2007, 56, 2225-2231. doi:10.1007/s11172-007-0348-x

22. Wang, Y.; Yuan, H.; Lu, H.; Zheng, W.-H. Org. Lett. 2018, 20 , 2555-2558. doi:10.1021/acs.orglett.8b00711

23. Hitchcock, P. B.; Rowlands, G. J.; Parmar, R. Chem. Commun. 2005, 4219-4221. doi:10.1039/b507394d

24. Parmar, R.; Coles, M. P.; Hitchcock, P. B.; Rowlands, G. J. Synthesis 2010, 4177-4187. doi:10.1055/s-0030-1258286

25. Cipiciani, A.; Fringuelli, F.; Mancini, V.; Piermatti, O.; Scappini, A. M.; Ruzziconi, R. Tetrahedron 1997, 53, 11853-11858. doi:10.1016/s0040-4020(97)00758-8

26. Pamperin, D.; Schulz, C.; Hopf, H.; Syldatk, C.; Pietzsch, M. Eur. J. Org. Chem. 1998, 1441-1445. doi:10.1002/(sici)1099-0690(199807)1998:7<1441::aid-ejoc1441>3.0.c o;2-k

27. Pamperin, D.; Ohse, B.; Hopf, H.; Pietzsch, M. J. Mol. Catal. B: Enzym. 1998, 5, 317-319. doi:10.1016/s1381-1177(98)00063-0

28. Mori, K.; Kishi, H.; Akiyama, T. Synthesis 2017, 49, 365-370. doi:10.1055/s-0036-1588898

29. Taylor, J. E.; Bull, S. D.; Williams, J. M. J. Chem. Soc. Rev. 2012, 41, 2109-2121. doi:10.1039/c2cs15288f

30. Merad, J.; Pons, J.-M.; Chuzel, O.; Bressy, C. Eur. J. Org. Chem. 2016, 5589-5610. doi:10.1002/ejoc.201600399

31. Birman, V. Aldrichimica Acta 2016, 49, 23-33.

32. McLaughlin, C.; Smith, A. D. Chem. - Eur. J. 2021, 27, 1533-1555. doi:10.1002/chem.202002059

33. Birman, V. B.; Li, X. Org. Lett. 2006, 8, 1351-1354. doi:10.1021/ol060065s

34. Birman, V. B.; Li, X. Org. Lett. 2008, 10, 1115-1118. doi:10.1021/ol703119n

35. Li, X.; Jiang, H.; Uffman, E. W.; Guo, L.; Zhang, Y.; Yang, X.; Birman, V. B. J. Org. Chem. 2012, 77, 1722-1737. doi:10.1021/jo202220x
36. Qu, S.; Greenhalgh, M. D.; Smith, A. D. Chem. - Eur. J. 2019, 25 , 2816-2823. doi:10.1002/chem.201805631

37. Qu, S.; Smith, S. M.; Laina-Martín, V.; Neyyappadath, R. M.; Greenhalgh, M. D.; Smith, A. D. Angew. Chem., Int. Ed. 2020, 59, 16572-16578. doi:10.1002/anie.202004354

38. Joannesse, C.; Johnston, C. P.; Concellón, C.; Simal, C.; Philp, D.; Smith, A. D. Angew. Chem., Int. Ed. 2009, 48, 8914-8918. doi:10.1002/anie.200904333

39. Cipiciani, A.; Fringuelli, F.; Mancini, V.; Piermatti, O.; Pizzo, F.; Ruzziconi, R. J. Org. Chem. 1997, 62, 3744-3747. doi:10.1021/jo962142a

40. Kagan, H. B.; Fiaud, J. C. Top. Stereochem. 1988, 18, 249-330. doi:10.1002/9780470147276.ch4

41. Greenhalgh, M. D.; Taylor, J. E.; Smith, A. D. Tetrahedron 2018, 74, 5554-5560. doi:10.1016/j.tet.2018.05.069

42. Calculation from recovered $2: s=\ln [(1-C)(1-\mathrm{ee}(2))] / \ln [(1-C)(1+$ ee(2))].

43. Calculation from isolated $3: s=\ln [1-C(1+\mathrm{ee}(3))] / \ln [1-C(1-$ $\mathrm{ee}(\mathbf{3}))$ ].

\section{License and Terms}

This is an Open Access article under the terms of the Creative Commons Attribution License (https://creativecommons.org/licenses/by/4.0). Please note that the reuse, redistribution and reproduction in particular requires that the author(s) and source are credited and that individual graphics may be subject to special legal provisions.

The license is subject to the Beilstein Journal of Organic Chemistry terms and conditions: (https://www.beilstein-journals.org/bjoc/terms)

The definitive version of this article is the electronic one which can be found at: https://doi.org/10.3762/bjoc. 17.68 